\title{
Theoretical discussion on reduced aberration sensitivity of enhanced alternating phase-shifting masks
}

\author{
Alfred K. Wong \\ Department of Electrical and Electronic Engineering \\ The University of Hong Kong, Pokfulam Road, Hong Kong
}

\begin{abstract}
Two theories are developed to quantify image skew of photomask features caused by aberrations. In one formulation, the extent of image distortion can be described by the image asymmetry $\left(\Delta \hat{x}_{\text {asymmetry }}\right)$, which captures both image shift and sidelobe intensity imbalance. This quantity is equivalent to the shift of the image centroid. In situations where one is more concerned with placement error than centroid shift, the change in the location of the intensity extremum $\left(\Delta \hat{x}_{\text {shift }}\right)$ can be expressed as functions of the mask spectrum and the wave aberration. This theory on image shift is applied to the study of enhanced alternating PSMs. Although the optimal mask pattern is aberration-function-specific, mask spectra with gradual variations have lower placement sensitivity in general. These theories are applicable to all mask technologies and patterns.
\end{abstract}

Keywords: optical lithography, phase-shifting mask, aberrations, alternating phase-shifting mask, enhanced alternating phase-shifting mask

\section{INTRODUCTION}

Resolution enhancement techniques ${ }^{1}$ such as modified illumination, ${ }^{2-5}$ assist features ${ }^{6,7}$ and phase-shifting masks (PSMs) ${ }^{8-10}$ are becoming indispensable optical lithography methods for the fabrication of integrated circuits. Some of these techniques are generally applicable, while others are beneficial only for specific pattern configurations. For the printing of small, dark features, alternating PSMs offer robust imaging because of destructive interference between light rays that are $180^{\circ}$ out of phase. This property and its promise for gatelevel applications have prompted studies by many authors. ${ }^{11-15}$ Linewidths as small as $k_{1}=0.1^{16}$ have been demonstrated.

Despite superior image quality, alternating PSMs are not amenable to straightforward implementation. Issues include intensity imbalance due to glass edge scattering, ${ }^{17-19}$ mask inspection and repair, ${ }^{20,21}$ aberration sensitivity, ${ }^{2-24}$ and the need for sophisticated computer-aided design (CAD) algorithms. ${ }^{25}$ In addition, the preference for lower-partial-coherence-factor (low- $\sigma$ ) exposure means that proximity effects are significant. Coupled with small mask error factors, ${ }^{26,27}$ optical proximity correction (OPC) by chromium width biasing is challenging.

Proximity correction can be achieved by modifying the widths of the phase regions (hereafter as phase width) on an alternating PSM. Another situation where there is a need for phase width adjustment is the balancing of intensity between the $0^{\circ}$ and $180^{\circ}$ openings. ${ }^{19,28}$ Beyond these two considerations, the phase regions are usually designed to be as wide as possible for maximum process latitude. Figure 1 plots the simulated exposure latitude of a $0.3(\lambda / \mathrm{NA})$ line as a function of the phase width. As the phase width increases from $0.3(\lambda / \mathrm{NA})$, the exposure latitude steadily increases from $20 \%$, reaching an asymptotic value of $33 \%$ for phase widths greater than $0.8(\lambda / \mathrm{NA})$.

Widening the phase regions is not always advantageous. It was recently reported that image sensitive to aberrations increases with phase width, while the use of small phase regions can reduce aberration sensitivity by over $70 \%{ }^{29}$ There thus exists a tradeoff between exposure latitude and low aberration sensitivity. Optimization results indicated that the optimum number of auxiliary phase regions is 1 or 2 per line edge, and their phase widths should be between $0.2(\lambda / \mathrm{NA})$ and $0.5(\lambda / \mathrm{NA}) .{ }^{29}$ The structure of these so-called enhanced alternating PSMs is shown in Fig. 2. This study aims to investigate the reason for the reduced aberration sensitivity of enhanced alternating PSMs.

Further author information: awong@eee.hku.hk 

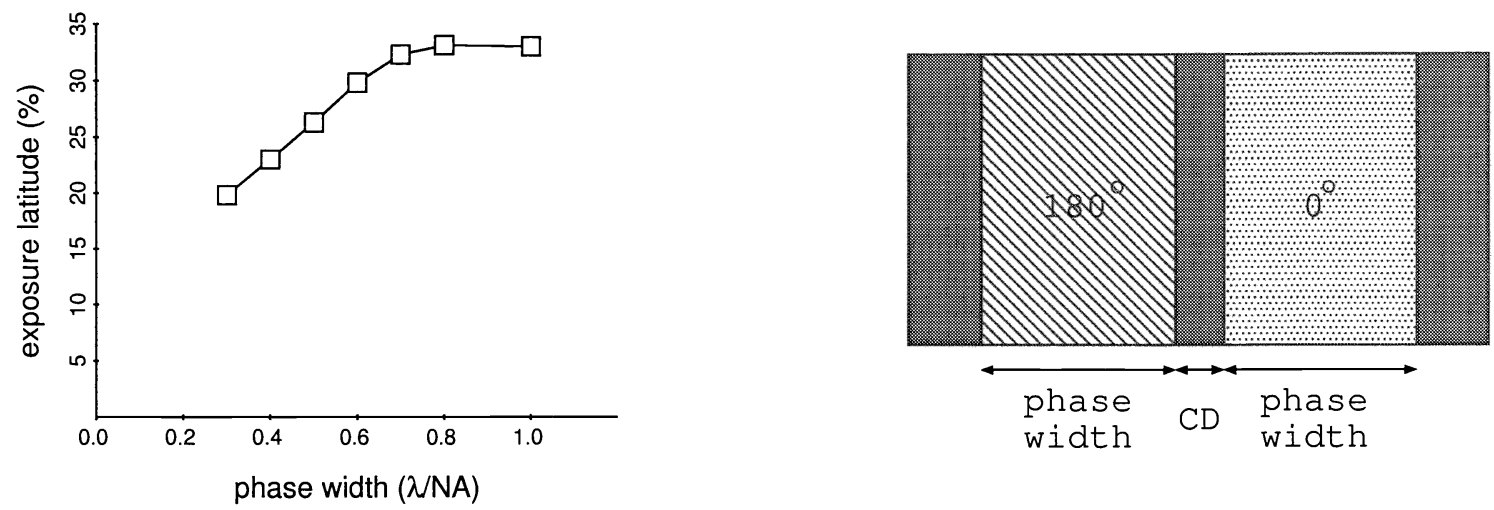

Figure 1. Exposure latitude of lines printed with alternating PSMs increases with phase width. The images were simulated with $\sigma=0.5$.

\section{IMAGE ASYMMETRY}

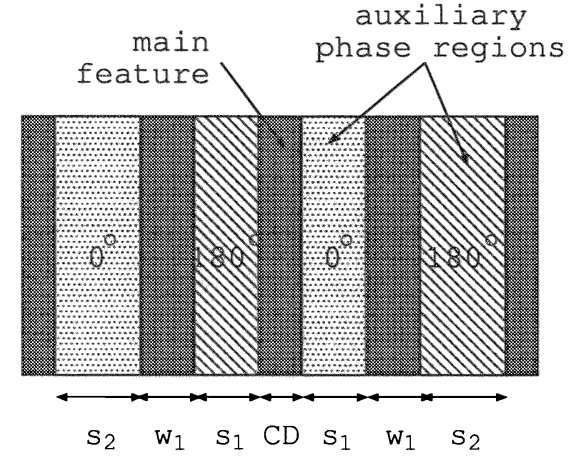

Figure 2: Structure of an enhanced alternating PSM.

We first examine image asymmetry caused by aberrations. Consider the much-simplified optical imaging process, as shown in Fig. 3. The image of an object point $P_{0}^{\mathrm{obj}}$ (not shown in the figure) is formed by the interference of light waves propagating from the exit pupil to the image plane. In the absence of aberrations, the wavefront of these light rays forms a spherical surface $S$ at the exit pupil. This surface $S$ is called the Gaussian reference sphere; it is centered at the Gaussian image point $P_{0}=\left(x_{0}, y_{0}\right)$ with a radius of curvature $R$.

Now consider an aberrated wavefront, represented by the surface $W$ in Fig. 3. The image forming rays of this aberrated system no longer converge at the Gaussian image point $P_{0}$. If we pick an arbitrary ray at $\left(f^{\prime}, g^{\prime}\right)$ in the exit pupil, it would intersect the image plane at $P_{0}^{\prime}=\left(x^{\prime}, y^{\prime}\right)$, a point that is most likely different from $P_{0}=\left(x_{0}, y_{0}\right)$. Denoting the optical path difference between the aberrated wavefront $W$ and the Gaussian reference sphere $S$ by the wave aberration $\Phi$, the displacement of $P_{0}^{\prime}$ from the Gaussian image point, or the ray aberration as it is commonly called, is ${ }^{30}$

$$
\left(x^{\prime}-x_{0}, y^{\prime}-y_{0}\right)=\left.(\Delta x, \Delta y)\right|_{\left(f^{\prime}, g^{\prime}\right)}=\left.\frac{R}{n}\left(\frac{\partial \Phi(f, g)}{\partial f}, \frac{\partial \Phi(f, g)}{\partial g}\right)\right|_{\left(f=f^{\prime}, g=g^{\prime}\right)},
$$

where $n$ is the refractive index of the image space. The ray aberration at $\left(f^{\prime}, g^{\prime}\right)$ is thus proportional to the gradient of the wave aberration at that pupil location.

In projection photolithography with a wavelength of $\lambda$ and a numerical aperture of NA, we can rewrite Eq. (1), normalizing spatial frequency variables to units of $(\mathrm{NA} / \lambda)$ and spatial dimensions to units of $(\lambda / \mathrm{NA})$ :

$$
\begin{aligned}
\left.(\Delta x, \Delta y)\right|_{\left(\hat{f}^{\prime}, \hat{g}^{\prime}\right)} & =\left.\frac{\lambda}{\mathrm{NA}}\left(\frac{\partial \Phi(\hat{f}, \hat{g})}{\partial \hat{f}}, \frac{\partial \Phi(\hat{f}, \hat{g})}{\partial \hat{g}}\right)\right|_{\left(\hat{f}=\hat{f}^{\prime}, \hat{g}=\hat{g}^{\prime}\right)}, \\
\left.(\Delta \hat{x}, \Delta \hat{y})\right|_{\left(\hat{f}^{\prime}, \hat{g}^{\prime}\right)} & =\left.\left(\frac{\partial \Phi(\hat{f}, \hat{g})}{\partial \hat{f}}, \frac{\partial \Phi(\hat{f}, \hat{g})}{\partial \hat{g}}\right)\right|_{\left(\hat{f}=\hat{f}^{\prime}, \hat{g}=\hat{g}^{\prime}\right)} .
\end{aligned}
$$

In Eq. (2), the symbol ^ represents normalized quantities, and imaging in air is assumed $(n=1)$. Using this equation, we can estimate the asymmetry of an image caused by aberrations. Let us examine coherent imaging 


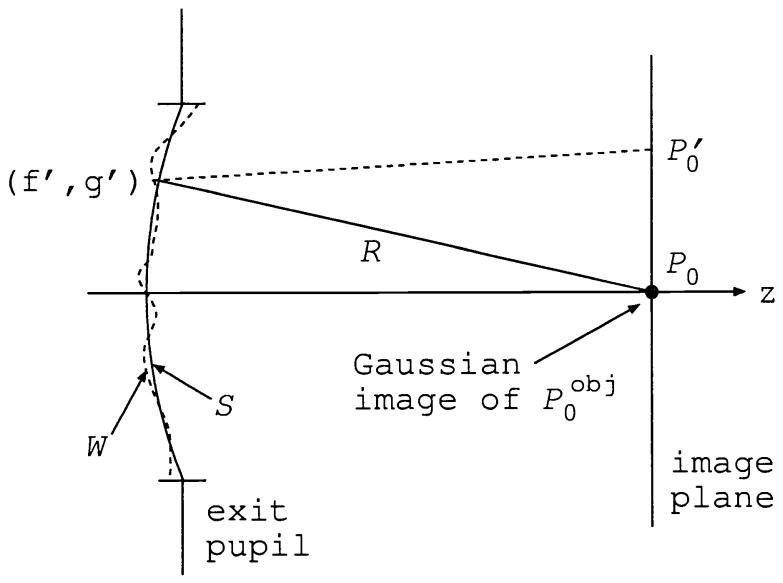

Figure 3. The ray aberration is proportional to the gradient of the wave aberration.

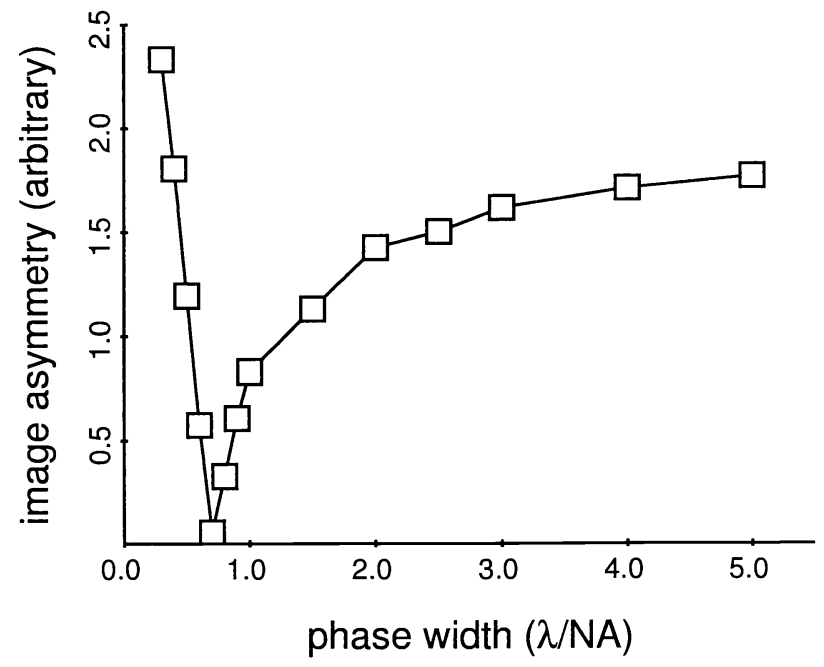

Figure 4. The degree of image asymmetry increases as the phase width deviates from $0.7(\lambda / N A)$.

of a one-dimensional feature. Suppose, without loss of generality, that the pattern varies only in the $x$-direction, the field distribution in the exit pupil plane is then the spectrum of the object $\tilde{\hat{O}}_{x}\left(\hat{f}^{\prime}\right)$. The ray aberration of the frequency component $\hat{f}^{\prime}$ is

$$
\Delta \hat{x}=\left.\frac{\partial \Phi(\hat{f}, \hat{g})}{\partial \hat{f}}\right|_{\left(\hat{f}=\hat{f}^{\prime}, \hat{g}=0\right)},
$$

and the amount of redirected energy is $\left|\tilde{\hat{O}}_{x}\left(\hat{f}^{\prime}\right)\right|^{2}$. The contribution of this ray at $\hat{f}=\hat{f}^{\prime}$ to the total image asymmetry is therefore

$$
\left.\frac{\left|\tilde{\hat{O}}_{x}\left(\hat{f}^{\prime}\right)\right|^{2}}{\int_{-1}^{+1}\left|\tilde{\hat{O}}_{x}(\hat{f})\right|^{2} d \hat{f}} \frac{\partial \Phi(\hat{f}, \hat{g})}{\partial \hat{f}}\right|_{\left(\hat{f}=\hat{f}^{\prime}, \hat{g}=0\right)}=\left.\frac{\left|\tilde{\hat{O}}_{x}\left(\hat{f}^{\prime}\right)\right|^{2}}{E_{\text {total }}} \frac{\partial \Phi(\hat{f}, \hat{g})}{\partial \hat{f}}\right|_{\left(\hat{f}=\hat{f}^{\prime}, \hat{g}=0\right)}
$$

where $E_{\text {total }}=\int_{-1}^{+1}\left|\tilde{\hat{O}}_{x}(\hat{f})\right|^{2} d \hat{f}$ is the total energy of all image forming rays. Integrating Eq. (3) over the exit pupil results in the image asymmetry:

$$
\Delta \hat{x}_{\text {asymmetry }}=\left.\frac{1}{E_{\text {total }}} \int_{-1}^{+1}\left|\tilde{\hat{O}}_{x}(\hat{f})\right|^{2} \frac{\partial \Phi\left(\hat{f}^{\prime \prime}, \hat{g}^{\prime \prime}\right)}{\partial \hat{f}^{\prime \prime}}\right|_{\left(\hat{f}^{\prime \prime}=\hat{f}, \hat{g}^{\prime \prime}=0\right)} d \hat{f} .
$$

Let us now apply Eq. (4) to alternating PSMs with one phase region per edge. With a wave aberration of $\Phi(\hat{\rho}, \phi)=\left(3 \hat{\rho}^{3}-2 \hat{\rho}\right) \cos \phi$ (balanced third-order coma), ${ }^{31}$ the image asymmetry of a $0.3(\lambda / \mathrm{NA})$ line as a function of the phase width is plotted in Fig. 4. The degree of asymmetry is the least for a phase width of $0.7(\lambda / \mathrm{NA})$; it increases rapidly as the phase width deviates from this value.

To understand the increased image asymmetry as the phase width deviates from $0.7(\lambda / \mathrm{NA})$, it is useful to express the spectrum of an alternating PSM as functions of the critical dimension $\hat{C D}$ and the phase width $\hat{s}$ :

$$
\tilde{\hat{O}}_{x}(\hat{f})=\frac{i 2}{\pi \hat{f}} \sin (\pi \hat{f} \hat{s}) \sin [\pi \hat{f}(\hat{C D}+\hat{s})] .
$$




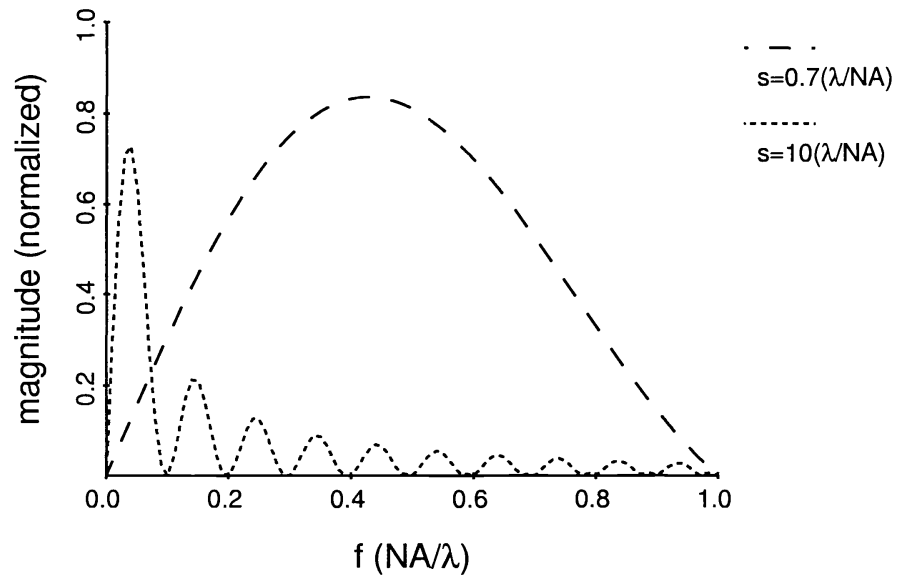

(a)

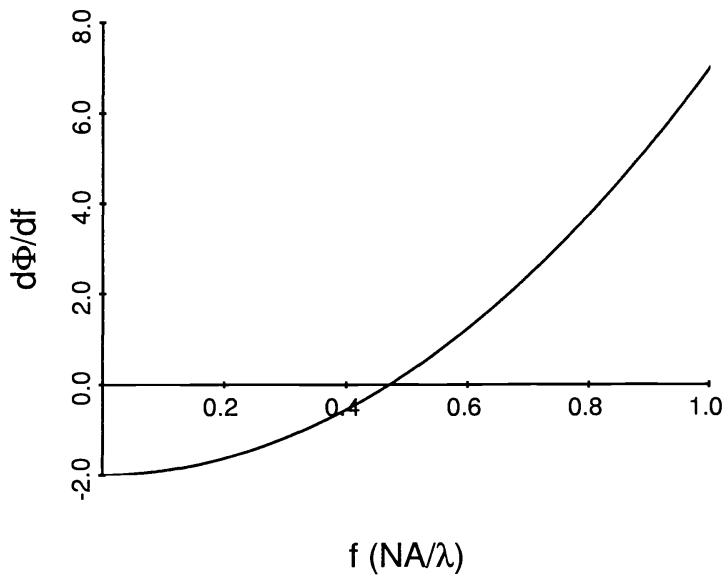

(b)

Figure 5. (a) Spectra of alternating PSMs with different phase widths show marked differences. The mask with the smaller phase width $[0.7(\lambda / N A)]$ exhibits a more gradual behavior than the one with the larger phase width $[10(\lambda / N A)]$. (b) The ray aberration of $\Phi(\hat{\rho}, \phi)=\left(3 \hat{\rho}^{3}-2 \hat{\rho}\right) \cos \phi$ in the $x$-direction.

Let us compare the spectra of alternating PSMs with the same CD of $0.3(\lambda / \mathrm{NA})$, but with two different phase widths of $0.7(\lambda / \mathrm{NA})$ and $10(\lambda / \mathrm{NA})$. Plotted in Fig. 5(a), the spectra of the two alternating PSMs show marked differences. For the mask with a phase width of $0.7(\lambda / \mathrm{NA})$, the spectrum magnitude initially increases gradually with frequency, reaching a maximum at a frequency of $\hat{f}=0.43$ before slowly decreasing to zero at $\hat{f}=1.0$. The spectrum of the mask with $\hat{s}=10$, on the other hand, shows a highly oscillatory behavior. There are more than 10 local maxima within the frequency range from $0-1(\lambda / \mathrm{NA})$. These peaks decrease in value with increasing frequency, with the largest value of 0.728 at $\hat{f}=0.04$.

The more gradual spectrum of the mask with a phase width of $0.7(\lambda / \mathrm{NA})$ results in lower sensitivity to aberrations. This can be understood with reference to Fig. $5(\mathrm{~b})$, which plots the ray aberration of $\Phi(\hat{\rho}, \phi)=$ $\left(3 \hat{\rho}^{3}-2 \hat{\rho}\right) \cos \phi$ in the $x$-direction as a function of the spatial frequency. As the frequency increases from zero, the gradient increases quadratically from -2 , reaching a value of 7 at $\hat{f}=1$. The zero crossing occurs at $\hat{f}=0.471$. Image forming rays corresponding to frequencies less than $\hat{f}=0.471$ are shifted in one direction, whereas rays whose freqencies are larger than $\hat{f}=0.471$ are shifted in the opposite direction.

For a phase width of $0.7(\lambda / \mathrm{NA})$, the spectrum peaks at $\hat{f}=0.43$, around spatial frequencies where the ray aberration has low values. The quantity $\left.\left|\tilde{\hat{O}}_{x}\left(\hat{f}^{\prime}\right)\right|^{2} \frac{\partial \Phi(\hat{f}, \hat{g})}{\partial \hat{f}}\right|_{(\hat{f}=\hat{f}, \hat{g}=0)}$ is therefore small. Further, the gradual variation of the spectrum results in more extensive averaging of energy shifts in the positive and negative directions. On the other hand, for an alternating PSM with a wide phase region such as a phase width of $10(\lambda / \mathrm{NA})$ in our example, the spectrum is dominated by the frequencies around $0.04(\mathrm{NA} / \lambda)$. Unless this dominant frequency coincides with a zero of the ray aberration function, there is less averaging across the exit pupil, and hence more image asymmetry.

\section{IMAGE SHIFT}

From wave-diffraction optics considerations, $\Delta \hat{x}_{\text {asymmetry }}$ computed from Eq. (4) is equivalent to the centroid of the image ${ }^{32}$ :

$$
\Delta \hat{x}_{\text {asymmetry }}=<\hat{x}>=\int_{-\infty}^{+\infty} \hat{x} \hat{I}_{x}(\hat{x}) d \hat{x} .
$$




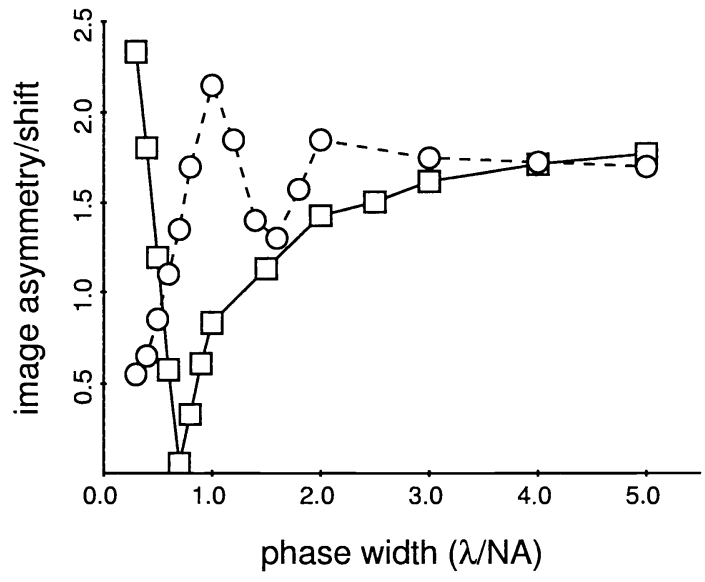

(a)

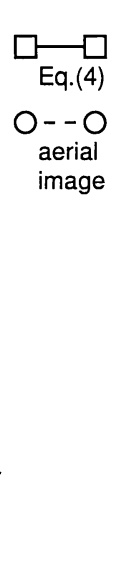

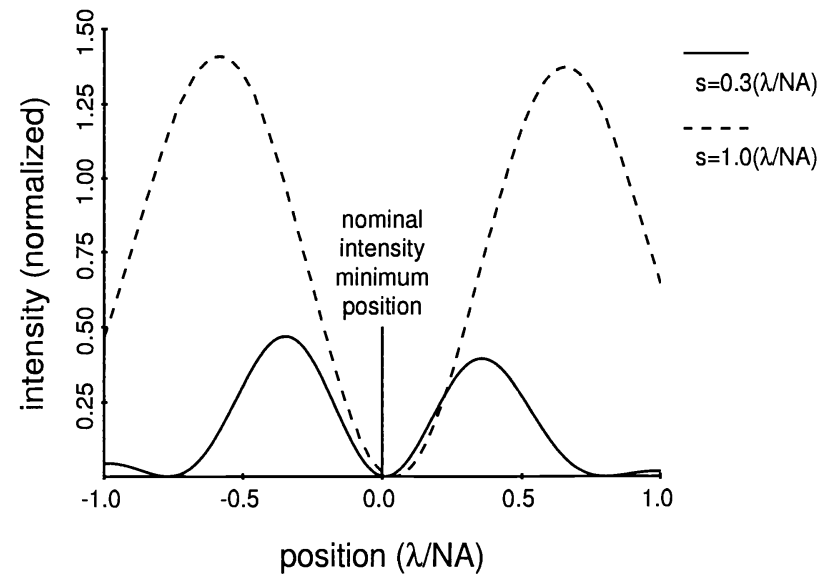

(b)

Figure 6. (a) Comparison between $\Delta \hat{x}_{\text {asymmetry }}$ computed from Eq. (4) and image shift derived from aerial image simulation. The CD is $0.3(\lambda / N A)$. (b) Simulated aerial images of alternating PSMs with 2 different phase widths. Asymmetry of masks with small phase widths manifests mainly as an imbalance of the sidelobe intensities, while that of large-phase-width masks is primarily a result of image placement error.

Equation(4) is thus a signification of the image centroid shift rather than a direct measure of the shift in the location of the intensity extremum (hereafter as image shift). The difference is illustrated in Fig. 6(a), which compares the image asymmetry as computed from Eq. (4) [Fig. 4 repeated as the curve with square markers] with the image shift derived from aerial image simulation (curve with circular markers). Except for large phase widths, Eq. (4) is not a good indicator of the image shift. The discrepancy arises because two phenomena contribute to image asymmetry: image placement error and sidelobe intensity imbalance, and Eq. (4) captures both. For phase widths smaller than $0.7(\lambda / \mathrm{NA})$, image asymmetry manifests mainly as an imbalance of the sidelobe intensities, as shown by the solid curve in Fig. 6(b). In this case, a large image skew does not translate to a large placement error. For large phase widths, on the other hand, the high degree of image asymmetry is primarily the result of placement error of the image [the dotted curve in Fig. 6(b)].

For certain lithography applications such as the gate level, one is often more concerned with image shift rather than intensity imbalance of the bright regions. For well-corrected exposure systems in which the Strehl ratios $^{33}$ are close to unity, image placement error is virtually identical to the image shift. To develop a theory relating image shift with aberrations, let us first consider a 2-beam imaging scenario. The imaging field is given by the sum of two plane waves with amplitudes $\tilde{\hat{O}}_{x}\left(\hat{f}_{1}\right)$ and $\tilde{\hat{O}}_{x}\left(\hat{f}_{2}\right)$ :

$$
\begin{aligned}
E_{\text {field }} & =\tilde{\hat{O}}_{x}\left(\hat{f}_{1}\right) e^{i 2 \pi\left(\hat{f}_{1} \hat{x}+\phi_{1}\right)}+\tilde{\hat{O}}_{x}\left(\hat{f}_{2}\right) e^{i 2 \pi\left(\hat{f}_{2} \hat{x}+\phi_{2}\right)} \\
& =e^{i 2 \pi \frac{\hat{f}_{1} \phi_{2}-\hat{f}_{2} \phi_{1}}{\hat{f}_{1}-\hat{f}_{2}}}\left[\tilde{\hat{O}}_{x}\left(\hat{f}_{1}\right) e^{i 2 \pi \hat{f}_{1}\left(\hat{x}+\frac{\phi_{1}-\phi_{2}}{\hat{f}_{1}-\hat{f}_{2}}\right)}+\tilde{\hat{O}}_{x}\left(\hat{f}_{2}\right) e^{i 2 \pi \hat{f}_{2}\left(\hat{x}+\frac{\phi_{1}-\phi_{2}}{\hat{f}_{1}-\hat{f}_{2}}\right)}\right],
\end{aligned}
$$

where $\phi_{i}=\Phi\left(\hat{f}_{i}, 0\right)$. The image shift is thus

$$
\Delta \hat{x}_{\text {shift }}=-\frac{\phi_{1}-\phi_{2}}{\hat{f}_{1}-\hat{f}_{2}}
$$

In this situation, we can interpret the factor $\left(\frac{\phi_{1}-\phi_{2}}{\hat{f}_{1}-\hat{f}_{2}}\right)$ as the discrete ray aberration. The image shift is solely dependent on the discrete gradient of the wave aberration.

Let us now extend our analysis to a 3-beam imaging scenario with the object spectrum

$$
\tilde{\hat{O}}_{x}(\hat{f})=a_{1} \delta\left(\hat{f}-\hat{f}_{1}\right)+a_{2} \delta\left(\hat{f}-\hat{f}_{2}\right)+a_{3} \delta\left(\hat{f}-\hat{f}_{3}\right),
$$


where the $a_{i}$ s are the amplitudes of the diffraction orders, and $\delta($.$) is the Dirac delta function. { }^{34}$ The image intensity under coherent illumination is given by

$$
I(\hat{x})=\int_{-1}^{+1} \int_{-1}^{+1} \tilde{\hat{O}}_{x}\left(\hat{f}_{a}\right) \tilde{\hat{O}}_{x}^{*}\left(\hat{f}_{b}\right) e^{-i 2 \pi\left[\left(\hat{f}_{a}-\hat{f}_{b}\right) \hat{x}+\left(\phi_{a}-\phi_{b}\right)\right]} d \hat{f}_{a} d \hat{f}_{b}
$$

where the asterisk $*$ denotes complex conjugation. The intensity extremum can be computed by taking the derivative of Eq. (6) with respect to $\hat{x}$ :

$$
\begin{aligned}
& \frac{d I(\hat{x})}{d \hat{x}}=4 \pi\left[\Delta \hat{f}_{12} \operatorname{Im}\left(a_{1} a_{2}^{*} e^{-i 2 \pi\left(\Delta \hat{f}_{12} \hat{x}+\phi_{12}\right)}\right)\right.+ \\
&\left.\Delta \hat{f}_{23} \operatorname{Im}\left(a_{2} a_{3}^{*} e^{-i 2 \pi\left(\Delta \hat{f}_{23} \hat{x}+\phi_{23}\right)}\right)+\Delta \hat{f}_{31} \operatorname{Im}\left(a_{3} a_{1}^{*} e^{-i 2 \pi\left(\Delta \hat{f}_{31} \hat{x}+\phi_{31}\right)}\right)\right],
\end{aligned}
$$

where $\Delta \hat{f}_{i j}=\left(\hat{f}_{i}-\hat{f}_{j}\right), \phi_{i j}=\left(\phi_{i}-\phi_{j}\right)$, and $\operatorname{Im}(\cdot)$ represents the imaginary part of (.). Since the image shift $\Delta \hat{x}_{\text {shift }}$ as well as the $\phi_{i j}$ s are small compared with 1 in a typical lithography process, Eq. (7) can be approximated by

$$
\frac{d I(\hat{x})}{d \hat{x}}=8 \pi^{2}\left[a_{1} a_{2} \Delta \hat{f}_{12}\left(\Delta \hat{f}_{12} \hat{x}+\phi_{12}\right)+a_{2} a_{3} \Delta \hat{f}_{23}\left(\Delta \hat{f}_{23} \hat{x}+\phi_{23}\right)+a_{3} a_{1} \Delta \hat{f}_{31}\left(\Delta \hat{f}_{31} \hat{x}+\phi_{31}\right)\right]
$$

for masks with real transmission functions. Setting $\frac{d I(\hat{x})}{d \hat{x}}=0$ in Eq. (8) results in

$$
\Delta \hat{x}_{\text {shift }}=-\frac{a_{1} a_{2} \Delta \hat{f}_{12} \phi_{12}+a_{2} a_{3} \Delta \hat{f}_{23} \phi_{23}+a_{3} a_{1} \Delta \hat{f}_{31} \phi_{31}}{a_{1} a_{2}\left(\Delta \hat{f}_{12}\right)^{2}+a_{2} a_{3}\left(\Delta \hat{f}_{23}\right)^{2}+a_{3} a_{1}\left(\Delta \hat{f}_{31}\right)^{2}}
$$

Rather than the discrete ray aberration $\left(\phi_{i j} / \Delta \hat{f}_{i j}\right)$, the image shift is a function of the frequency and phase difference between the image forming rays. We can generalize Eq. (9) to an aperiodic object with the spectrum $\tilde{\hat{O}}_{x}(\hat{f})$ :

$$
\Delta \hat{x}_{\text {shift }}=-\frac{\int_{-1}^{+1} \int_{\hat{f}_{2}}^{+1} \tilde{\hat{O}}_{x}\left(\hat{f}_{1}\right) \tilde{\hat{O}}_{x}\left(\hat{f}_{2}\right)\left(\hat{f}_{1}-\hat{f}_{2}\right)\left(\phi_{1}-\phi_{2}\right) d \hat{f}_{1} d \hat{f}_{2}}{\int_{-1}^{+1} \int_{\hat{f}_{2}}^{+1} \tilde{\hat{O}}_{x}\left(\hat{f}_{1}\right) \tilde{\hat{O}}_{x}\left(\hat{f}_{2}\right)\left(\hat{f}_{1}-\hat{f}_{2}\right)^{2} d \hat{f}_{1} d \hat{f}_{2}} .
$$

To validate the accuracy of Eq. (10), the image shift of a $0.3(\lambda / \mathrm{NA})$ line as a function of the phase width is plotted as the curve with square markers in Fig. 7. This curve shows excellent agreement with the image placement error derived from aerial image simulation (curve with circular markers in Fig. 7). The slight discrepancy between the curves arises from errors introduced during interpolation of the aerial images.

Equipped with Eq. (10), we are ready to analyze aberration sensitivity of enhanced alternating PSMs. The mask spectrum is

$$
\begin{aligned}
\tilde{\hat{O}}_{x}(\hat{f}) & =\sum_{n=1}^{N}(-1)^{n+1} \frac{i 2}{\pi \hat{f}} \sin \left(\pi \hat{f} \hat{s}_{n}\right) \sin \left[\pi \hat{f}\left(\hat{d}_{n}+\hat{s}_{n}\right)\right], \quad \text { where } \\
\hat{d}_{n} & =\hat{d}_{n-1}+2 \hat{s}_{n-1}+2 \hat{w}_{n-1} \quad \text { with } \quad \hat{d}_{1}=\hat{C D}
\end{aligned}
$$

$\hat{s}_{n}$ and $\hat{w}_{n}$ are defined in Fig. 2 , and $N$ is the number of phase regions per edge. We focus on mask structures whose phase widths and separations between the phase regions have the same dimension, i. e., $\hat{s}_{1}=\hat{s}_{2}=\cdots=$ $\hat{s}_{N}=\hat{w}_{1}=\hat{w}_{2}=\cdots=\hat{w}_{N-1}=\hat{s}$. Figure 8 is a density plot of the image shift of a $0.3(\lambda / \mathrm{NA})$ line as functions of the phase width $\hat{s}$ and the number of phase regions $N$ for balanced third-order coma. Image shift generally 


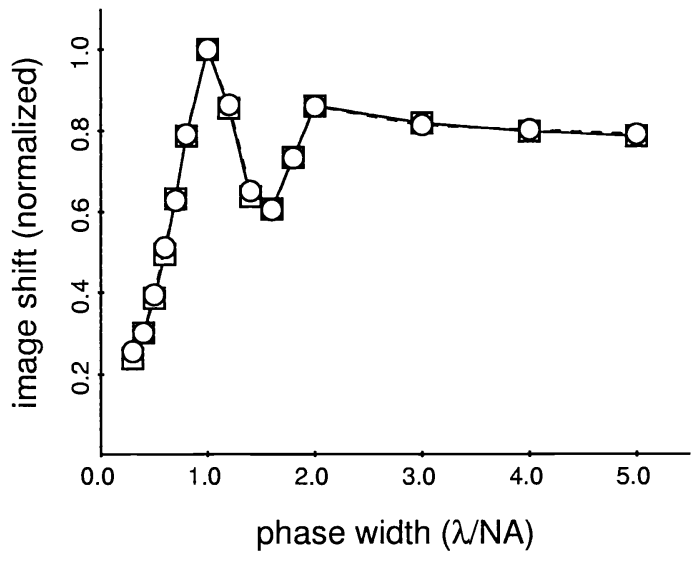

Figure 7. Comparison between $\Delta \hat{x}_{\text {shift }}$ computed from Eq. (10) and image placement error derived from aerial image simulation. The $C D$ is $0.3(\lambda / N A)$.

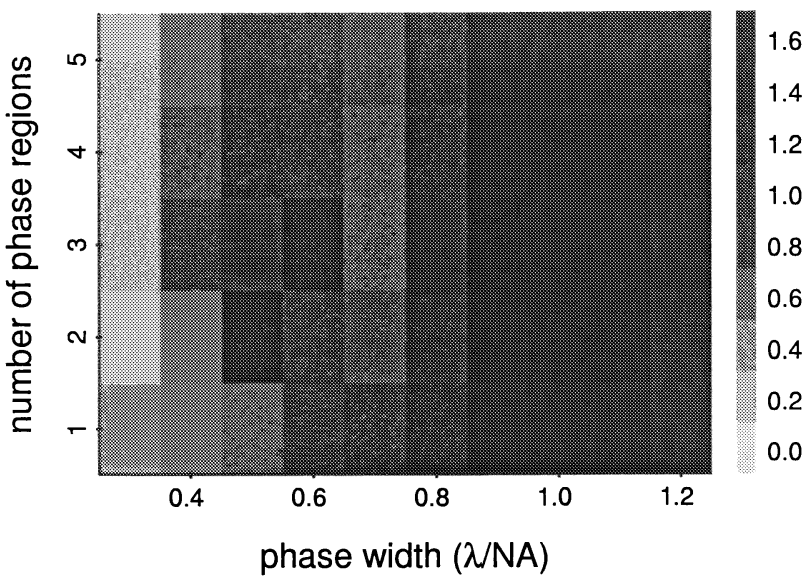

Figure 8. Image shift as functions of the phase width and the number of phase regions per edge for balanced thirdorder coma aberration $\left[\Phi(\hat{\rho}, \phi)=\left(3 \hat{\rho}^{3}-2 \hat{\rho}\right) \cos \phi\right]$.

increases with phase width, consistent with the data shown in Fig. 7. Regardless of the number of phase regions, the smallest phase width used in the study $[0.3(\lambda / \mathrm{NA})]$ has low aberration sensitivity. For a fixed phase width of $0.3(\lambda / \mathrm{NA})$, the image shift decreases as the number of phase regions is increased from 1 to 2 . However, subsequent increase in the number of phase regions worsens rather than improves the image shift.

To understand the interesting dependence of aberration sensitivity on the number of phase regions, let us examine the spectra of three enhanced alternating PSM with the same $\hat{C D}=0.3$ and $\hat{s}=0.3$, but with different numbers of phase regions per edge. In Fig. 9 , the spectrum of the mask with one phase region $(N=1)$ is plotted as the curve with alternating long and short dots. It has a gradual variation with frequency. Plotted in the same figure is the wave aberration of balanced third-order coma (solid curve). A study of these two curves provides a qualitative understanding of the image shift. To aid our analysis, let us divide the frequency space into 4 regions, as indicated in Fig. 9. The wave aberration has negative values in regions 1 and 3 , whereas $\Phi(\hat{f}, 0)$ assumes positive values in regions 2 and 4 . The image shift is proportional to the sum of the interactions between these regions according to the numerator of Eq. (10). The auto-interaction of region 1 (interaction between region 1 and itself) is not a major contributor to the image shift because both $\Delta \hat{f}_{i j}$ and the size of the region are small. The interaction between regions 1 and 2 ,

$$
\Delta_{12}=\int_{\text {region } 2} \int_{\text {region } 1} \tilde{\hat{O}}_{x}\left(\hat{f}_{1}\right) \tilde{\hat{O}}_{x}\left(\hat{f}_{2}\right)\left(\hat{f}_{1}-\hat{f}_{2}\right)\left(\phi_{1}-\phi_{2}\right) d \hat{f}_{1} d \hat{f}_{2},
$$

is significant because $\tilde{\hat{O}}_{x}\left(\hat{f}_{1}\right), \tilde{\hat{O}}_{x}\left(\hat{f}_{2}\right)$, and $\Delta \hat{f}_{12}$ are all sizable, and the sign of $\phi_{12}$ is constant (negative) throughout the integration regions. However, $\Delta_{12}$ is mostly canceled by $\Delta_{14}$, in which the product of $\tilde{\hat{O}}\left(\hat{f}_{1}\right)$ (positive), $\tilde{\hat{O}}\left(\hat{f}_{4}\right)$ (negative), and $\phi_{12}$ (negative) results in a shift in the opposite direction. Similar considerations for interactions between other regions lead to the conclusion that $\Delta_{23}$ is the dominant contributor to image shift because of the sizes of regions 2 and 3 , as well as the consistency in the signs of all the terms in the product $\tilde{\hat{O}}_{x}\left(\hat{f}_{2}\right) \tilde{\hat{O}}_{x}\left(\hat{f}_{3}\right) \Delta \hat{f}_{23} \phi_{23}$.

Repeating the procedure for an enhanced alternating PSM with 2 phase regions $(N=2)$ reveals that no single interaction term dominates. With the spectrum plotted as the curve with long dots in Fig. 9, the contribution to image shift from $\Delta_{23}$ is subdued (compared with the mask with 1 phase region per edge) because the spectrum amplitude changes sign within both regions 2 and 3 , leading to some amount of averaging of positive and negative shifts. The resulting $\Delta_{23}$ happens to be of similar magnitude to $\Delta_{14}$, but of a different sign. Since only these 2 interaction terms are significant, they almost cancel each other, leading to minimal image shift. 


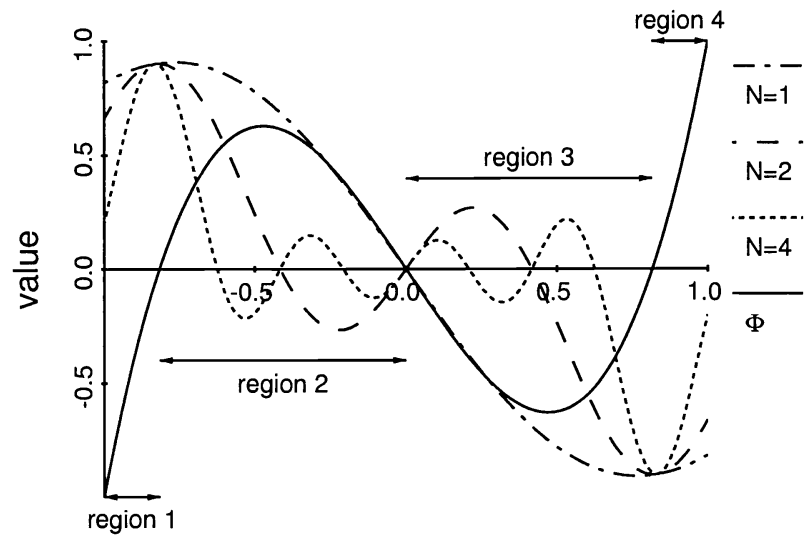

$f(N A / \lambda)$

Figure 9. Frequency spectra of enhanced alternating PSMs with different numbers of phase regions per edge.

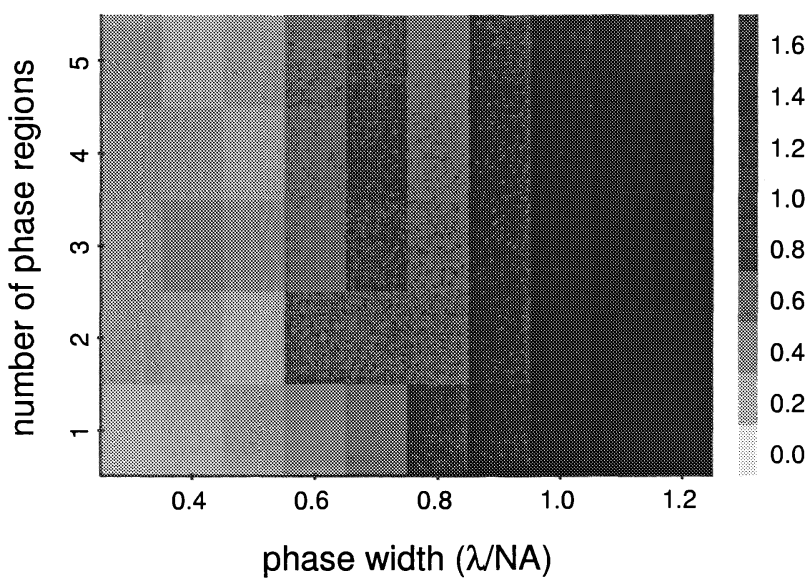

Figure 10. Image shift as functions of the phase width and the number of phase regions per edge for balanced fifth-order coma aberration $\left[\Phi(\hat{\rho}, \phi)=\left(10 \hat{\rho}^{5}-12 \hat{\rho}^{3}+3 \hat{\rho}\right) \cos \phi\right]$.

For a mask with 4 phase regions (curve with short dots in Fig. 9), $\Delta_{23}$ is further subdued because of the increased oscillation and the reduced magnitude of the spectrum within regions 2 and 3 . The interaction between regions 1 and 4 becomes the single dominant contributor to image shift, resulting in an increased $\Delta \hat{x}_{\text {shift }}$ compared with the mask with 2 phase regions.

\section{DISCUSSION}

The foregoing analysis indicates that the placement sensitivity to aberration of a mask feature is dependent on the wave aberration of the exposure system. One pattern may have lower sensitivity to balanced third-order coma than a second pattern; but the relative sensitivity may reverse for other aberration functions. For example, Fig. 10 is a density plot of the image shift of a $0.3(\lambda / \mathrm{NA})$ line as functions of the phase width and the number of phase regions per edge for balanced fifth-order coma aberration $\left.\left[\Phi(\hat{\rho}, \phi)=\left(10 \hat{\rho}^{5}-12 \hat{\rho}^{3}+3 \hat{\rho}\right) \cos \phi\right)\right]$. Although aberration sensitivity in general decreases with decreasing phase width, a phenomenon similar to third-order coma aberration (Fig. 8), the mask with $\hat{s}=0.3$ and $N=1$ has a lower aberration sensitivity than the one with $\hat{s}=0.3$ and $N=2$.

In the same vein, if the wave aberration of the exposure system is known, it is possible to optimize the enhanced alternating PSM structure for low placement sensitivity. For example, consider 2 aberration functions having the same peak and RMS values:

$$
\Phi_{1}(\hat{f}, 0)=\left\{\begin{array}{ll}
-\phi_{0} & -0.43 \leq \hat{f}<0, \\
\phi_{0} & 0<\hat{f} \leq 0.43, \\
0 & \text { otherwise }
\end{array} \quad \text { and } \quad \Phi_{2}(\hat{f}, 0)= \begin{cases}-\phi_{0} & -0.86 \leq \hat{f} \leq-0.43 \\
\phi_{0} & 0.43 \leq \hat{f} \leq 0.86 \\
0 & \text { otherwise }\end{cases}\right.
$$

In the imaging of a $0.3(\lambda / \mathrm{NA})$ line by an alternating PSM with a phase width of $0.7(\lambda / \mathrm{NA})$ [see Fig. $5(\mathrm{a})$ for its spectrum], the image shift is bigger for $\Phi_{2}(\hat{f}, 0)$ because the weighting factor $\left(\hat{f}_{1}-\hat{f}_{2}\right)$ of the phase difference $\left(\phi_{1}-\phi_{2}\right)$ is larger in Eq. (10). On the other hand, an alternating PSM with a wider phase width of $10(\lambda / N A)$ [its spectrum is shown in Fig. 5(a)] shifts more under the influence of $\Phi_{1}(\hat{f}, 0)$. The dominant image forming rays around $\hat{f}=0.04$ are aberrated by $\Phi_{1}(\hat{f}, 0)$ but not by $\Phi_{2}(\hat{f}, 0)$.

Although the placement sensitivity of a mask pattern is aberration-function-specific, we can still assert that a mask with a flatter spectrum in general has lower placement sensitivity than one whose spectrum is dominated by a few frequency components. Referring to the numerator of Eq. (10), a flatter spectrum typically results 
in a higher degree of cancellation between positive and negative shifts because the factor $\left(\phi_{1}-\phi_{2}\right)$ changes sign across the pupil. Compared with conventional alternating PSMs, enhanced alternating PSMs have lower placement sensitivity because their spectra exhibit a more gradual variation.

\section{SUMMARY}

Two theories for the quantification of image skew of photomask features caused by aberrations are developed. By summing the ray aberration weighted by the ray energy across the exit pupil, image asymmetry can be computed. This shift of the image centroid accounts for both image shift and sidelobe intensity imbalance. To isolate image placement error from the centroid shift, one needs to consider the shift of the intensity extremum. Application of the image shift formulation leads to the conclusion that enhanced alternating PSMs are generally less sensitive to aberrations because of their more gradual spectra compared with conventional alternating PSMs. Although applied only to enhanced alternating PSM in our discussion, the theories encapsulated by Eqs. (4) and (10) are generally applicable.

\section{REFERENCES}

1. A. K. Wong, Resolution Enhancement Techniques in Optical Lithography, SPIE Press, 2001.

2. K. Kamon, T. Miyamoto, Y. Myoi, H. Nagata, M. Tanaka, and K. Horie, "Photolithography system using annular illumination," Jpn. J. Appl. Phys. 30, pp. 3021-3029, Nov. 1991.

3. M. Noguchi, M. Muraki, Y. Iwasaki, and A. Suzuki, "Subhalf micron lithography system with phase-shifting effect," in Proc. SPIE, J. D. Cuthbert, ed., 1674, pp. 92-104, 1992.

4. H.-Y. Kang, C.-H. Kim, J.-H. Lee, W.-S. Han, and Y.-B. Koh, "High performance lithography with advanced modified illumination," IEEE Transactions on Electronics 77-C, pp. 432-437, Mar. 1994.

5. T. Ogawa, M. Uematsu, T. Ishimaru, and M. Kimura, "The effective light source optimization with the modified beam for the depth-of-focus enhancements," in Proc. SPIE, T. Brunner, ed., 2197, pp. 19-30, 1994.

6. M. D. Prouty and A. R. Neureuther, "Optical imaging with phase shift masks," in Proc. SPIE, 470, pp. 228-232, 1984.

7. J. Garofalo, C. Biddick, R. Kostelak, and S. Vaidya, "Mask assisted off axis illumination technique for random logic," J. Vac. Sci. Technol. B 11, pp. 2651-2658, Nov. 1993.

8. M. Levenson, N. Viswanathan, and R. Simpson, "Improving resolution in photolithography with a phaseshifting mask," IEEE Transactions on Electron Devices 29, pp. 1812-1846, Dec. 1982.

9. T. Terasawa, N. Hasegawa, and H. Fukuda, "Imaging characteristics of multi-phase-shifting and halftone phase-shifting masks," Jpn. J. Appl. Phys. 30, pp. 2991-2997, Nov. 1991.

10. B. J. Lin, "The attenuated phase-shifting mask," Solid State Technology 35, pp. 43-47, Jan. 1992.

11. T. Brunner, P. Sanda, M. Wordeman, and T. Lii, " $170 \mathrm{~nm}$ gates fabricated by phase-shift mask and top anti-reflector process," in Proc. SPIE, J. Cuthbert, ed., 1927, pp. 182-189, 1993.

12. C. A. Spence, "Mask for optical lithography using phase shift masking and integrated circuit produced therefrom." U.S. patent \#5702848, Dec. 1997.

13. H.-Y. Liu, L. Karklin, Y.-T. Wang, and Y. C. Pati, "The application of alternating phase-shifting masks to $140 \mathrm{~nm}$ gate patterning: Line width control improvements and design optimization," in Proc. SPIE, 3236, pp. 328-337, 1998.

14. H.-Y. Liu, L. Karklin, Y.-T. Wang, and Y. C. Pati, "Application of alternating phase-shifting masks to $140 \mathrm{~nm}$ gate patterning II: Mask design and manufacturing tolerances," in Proc. SPIE, 3334, pp. 2-14, 1998.

15. L. Liebmann, I. Graur, W. Leipold, J. Oberschmidt, D. O'Grady, and D. Regaill, "Alternating phase shifted mask for logic gate levels, design and mask manufacturing," in Proc. SPIE, L. van den Hove, ed., 3679, pp. 27-37, 1999 . 
16. S. Nakao, J. Itoh, A. Nakae, I. Kanai, T. Saitoh, H. Matsubara, K. Tsujita, I. Arimoto, and W. Wakamiya, "Extension of $\mathrm{KrF}$ lithography to sub-50 nm pattern formation," in Proc. SPIE, C. Progler, ed., 4000, pp. 358-365, 2000.

17. R. Kostelak, C. Pierrat, J. Garofalo, and S. Vaidya, "Exposure characteristics of alternate aperture phaseshifting masks fabricated using a subtractive process," J. Vac. Sci. Technol. B 10, pp. 3055-3061, Nov. 1992.

18. M. D. Levenson, "Phase shifting mask strategies: isolated dark lines," Microlithography World, pp. 6-12, Mar. 1992.

19. A. Wong and A. Neureuther, "Mask topography effects in projection printing of phase-shifting masks," IEEE Transactions on Electron Devices 41, pp. 895-902, June 1994.

20. J. N. Wiley, T. Y. Fu, T. Tanaka, S. Takeuchi, S. Aoyama, J. Miyazaki, and Y. Watakabe, "Phase shift mask pattern accuracy requirements and inspection technology," in Proc. SPIE, W. H. Arnold, ed., 1464, pp. 346-355, SPIE, 1991.

21. L. Liebmann, S. Mansfield, A. Wong, J. Smolinski, S. Peng, K. Kimmel, M. Rudzinski, J. Wiley, and L. Zurbrick, "High-resolution ultraviolet defect inspection of DAP (darkfield alternate phase) reticles," in Proc. SPIE, F. Abboud and B. Grenon, eds., 3873, pp. 148-161, 1999.

22. A. Nakae, S. Nakao, and Y. Matsui, "Proposal for pattern layout rule in application of alternating phaseshifting mask," in Proc. SPIE, N. Aizaki, ed., 3096, pp. 362-374, 1997.

23. S. Nakao, A. Nakae, K. Tsujita, and W. Wakamiya, "Impact of spherical aberrations on printing characteristics of irregularly aligned patterns of alternating phase shift mask," Jpn. J. Appl. Phys. 38, pp. 1919-1926, Apr. 1999.

24. R. Schenker, "Effects of phase shift masks on across field linewidth control," in Proc. SPIE, L. van den Hove, ed., 3679, pp. 18-26, 1999.

25. L. Liebmann, S. Mansfield, A. Wong, M. Lavin, W. Leipold, and T. Dunham, "TCAD/EDA development for lithography resolution enhancement," IBM J. Res. Develop. 45, pp. 651-665, Sept. 2001.

26. A. Wong, R. Ferguson, L. Liebmann, S. Mansfield, A. Molless, and M. Neisser, "Lithographic effects of mask critical dimension error," in Proc. SPIE, L. van den Hove, ed., 3334, pp. 106-116, 1998.

27. A. Wong, R. Ferguson, and S. Mansfield, "The mask error factor in optical lithography," IEEE Transactions on Semiconductor Manufacturing 13, pp. 235-242, May 2000.

28. R. Ferguson, L. Liebmann, S. Mansfield, D. O'Grady, and A. Wong, "Exact transmission balanced alternating phase-shifting mask for photolithography." U.S. patent \#5932377, Aug. 1999.

29. A. K. Wong, L. W. Liebmann, and A. F. Molless, "Alternating phase-shifting mask with reduced aberration sensitivity: lithography considerations," in Proc. SPIE, C. Progler, ed., 4346, pp. 420-428, 2001.

30. M. Born and E. Wolf, Principles of Optics, section 5.1, pp. 203-207. Pergamon Press, sixth ed., 1980.

31. V. N. Mahajan, "Zernike circle polynomials and optical aberrations of systems with circular pupils," Engineering \& Laboratory Notes, pp. S-21-S-24, Aug. 1994.

32. V. N. Mahajan, "Line of sight of an aberrated optical system," J. Opt. Soc. Am. A 2, pp. 833-846, June 1985.

33. J. W. Goodman, Introduction to Fourier Optics, chapter 6, p. 139. McGraw-Hill, 1968.

34. S. Gasiorowicz, Quantum Physics, appendix A, pp. 489-494. John Wiley \& Sons, 1974. 